\title{
Quadruple Attack of Gynecomastia
}

\author{
KARIMA ISMAIL, M.D.; MARIAM ISMAIL, M.D. and AHMED ISMAIL, M.D. \\ The Department of Plastic and Reconstructive Surgery, Faculty of Medicine, Cairo University
}

\begin{abstract}
Introduction: Gynecomastia is a common disorder. Its management presents several problems such as; excision of excess breast tissue, management of skin excess and the new position of the areola particularly in class II and III cases.
\end{abstract}

Material and Methods: This work presents a Quadruple attack to this problem which consists of liposuction, open excision of the glandular disc, undermining of inframammary fold and peri areolar skin excision. This technique was applied to 18 cases.

Results: Surgical follow-up varied from a minimum of 6 months to a maximum of 2 years, with an average of 1 year. With minimal complication rate $(11 \%)$ all of them are minor complications: Seroma, delayed wound healing and obvious scar. The aesthetic results were satisfactory to both patient and doctor.

Conclusion: Quadruple attack is a new surgical technique which is simple, reliable and easily attained by young surgeons. Nevertheless, it has good results with less complication rate.

Key Words: Quadruple attack - Gynecomastia.

\section{INTRODUCTION}

Gynecomastia is a common disorder; caused by increase in ductal tissue, stroma and/or fat in male breast, it may be associated with excess skin. Idiopathic is the most common cause; followed by obesity then secondary causes (systemic disorders, drugs). Nydick determined that there was an incidence of gynecomastia in $65 \%$ of pubertal males. Resolution almost always occurs but persists in small percentage [1]. In another study of 477 unselected autopsies, a $40 \%$ rate of gynecomastia was observed by Williams [2]. In a study of 306 adult males, clinical gynecomastia was seen in $36 \%$ with greater incidence $(57 \%)$ in men older than 44 years [3].
Gynecomastia was classified by Simon et al., into 3 grades [4]:

- Grade 1: Small enlargement, no skin excess.

- Grade 2A: Moderate enlargement, no skin excess.

- Grade 2B: Moderate enlargement with extra skin.

- Grade 3: Marked enlargement with extra skin.

On the other hand, Rohrich classified gynecomastia according to ultra sound assisted liposuction [5]:

- Grade I: Minimal hypertrophy (250g) without ptosis.

- Grade II: Moderate hypertrophy (250-500g) without ptosis.

- Grade III: Severe hypertrophy (500g) with grade 1 ptosis.

- Grade IV: Severe hypertrophy with grade 2 or 3 ptosis.

While Letterman and Schurter classified management of gynecomastia [6-8]:

- 1: Intra-areolar incision with no excess skin.

- 2: Intra-areolar incision with mild redundancy corrected with excision of skin through a superior peri areolar scar.

- 3: Excision of chest skin with or without shifting the nipple.

\section{MATERIAL AND METHODS}

Retrospective study included 23 cases, 18 cases were operated upon; 5 cases were observed. The treatment of gynecomastia should be aimed at 
correction of underlying cause. The pubertal male is most successfully treated with patience and reassurance. Thorough history taking, medical and laboratory evaluation should be done in order to exclude secondary causes.

Physical examination should enable the surgeon to determine the type of gynecomastia, Is it fibrous or fatty? Accordingly, the type of management is determined.

The management protocol was: Persistent gynecomastia less than 12 months and normal history and physical examination, observation and reassurance is recommended.

Persistent gynecomastia more than 12 months and normal history and physical examination, then surgical management is advised.

\section{Preparation:}

While the patient is standing identify the inframammary fold, pectoralis major muscle bulk, extent of gynecomastia and its type; whether fibrous or fatty or fibrofatty.

If there is significant breast enlargement and ptosis, identify the new nipple and areola complex position and approximate amount of skin excision. Mark the patient while standing.

All cases are done under general anesthesia.

\section{Surgical technique:}

Quadruple attack technique:

- Extensive liposuction.

- Excision of excess tissue.

- Extensive undermining.

- Excision of the skin.

\section{Extensive liposuction:}

Using tumescent technique, the standard wetting solution is: 1 liter of lactated ringer, $1 \mathrm{ml}$ epinephrine $(1: 1,000), 30 \mathrm{ml} \mathrm{1 \%} \mathrm{Xylocaine.} \mathrm{Wetting}$ solution is allowed 7 to 10 minutes for maximal vasoconstrictive effect.

Power assisted liposuction with cannula number 5 in deep levels above pectoralis major, cannula number 3 in superficial level.

\section{Excision of excess tissue:}

Through an inferior peri areolar incision, open resection or pull through of breast disc.

\section{Extensive undermining:}

Sharp dissection and release of inframammary fold. The fold is released mediallyup to $1 \mathrm{~cm}$ from midline; released laterally until the anterior axillary fold and released superiorly above the disc.

\section{Excision of the skin:}

First De-epithelialization of the distance for nipple elevation (superior pedicle) with preservation of $2 \mathrm{~cm}$ of de epithelialized tissue below areola.

Through Peri-areolar incision, concentric skin excision is done followed by Purse string sutures for closure, in 2 separate rings.

Drains are inserted.

\section{Post-operative care:}

Remove drains when it drains less than $40 \mathrm{~cm}$. Compressive garment is used continuously for first 3 weeks; the patient is allowed to remove the garment for a few minutes each day for bathing. Then followed by using it for only 12 hours for the following 3 months.

The patient applies antibiotic ointment to the suture lines twice per day for the first week. After 7 days the sutures are removed. During the first 24 hours the patient is encouraged to rest. After 48 hours, the patient may return to work. After 1 week the patient is encouraged to return to full activities including all sports. It is evident that those patients who return to full activities early heal much quicker and have resolution of edema, soreness, and ecchymosis much more rapidly. Sun exposure is avoided for 1 month.

\section{RESULTS}

We had no complications in $89 \%$ of the cases. We had Seroma in 1 case out of 18 cases (5.5\%). Delayed wound healing in 1 case $(5.5 \%)$ who was smoker and unsatisfactory scar in the same case.

Appearance of the scars were satisfactory to both doctor and the patient except 1 case $(5.5 \%)$ who complained of $2 \mathrm{~mm}$ widening of the scar whom was smoker. 


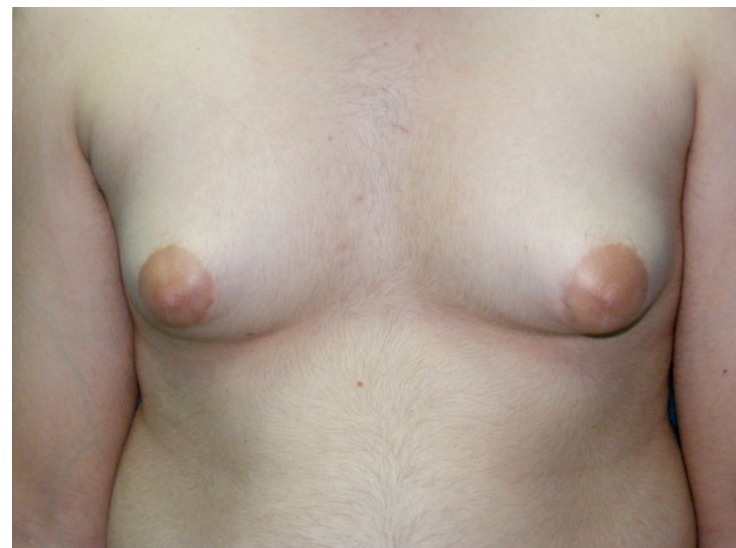

Fig. (1A): Pre-operative case of gynecomastia grade 3 .

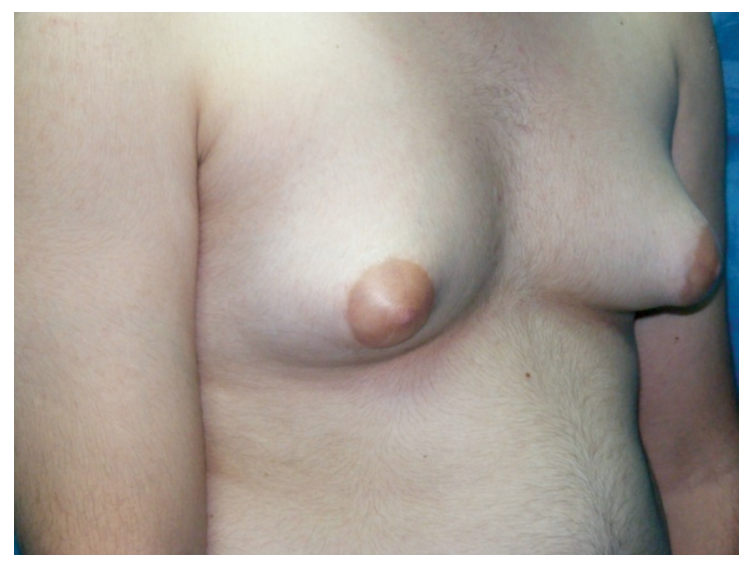

Fig. (2A): Pre-operative case of gynecomastia grade 3.

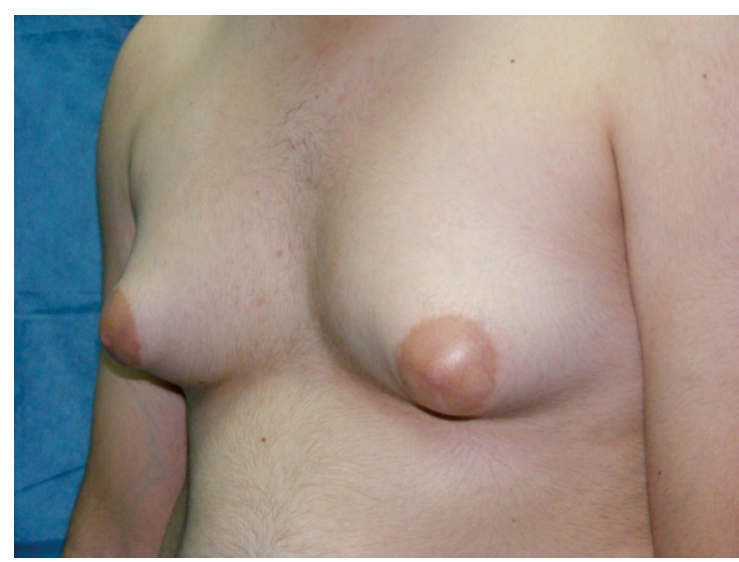

Fig. (3A): Pre-operative case of gynecomastia grade 3.

\section{DISCUSSION}

According to Simon classification and the type of gynecomastia parenchyma, the following is the most common used techniques:

\section{Fatty Gynecomastia:}

-1, 2a: Liposuction of the breast with tumescent rate $(1: 1)$.

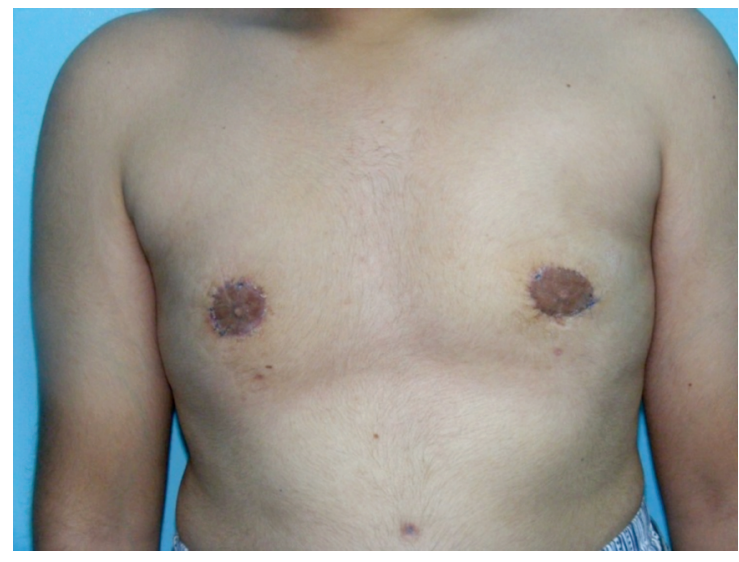

Fig. (1B): Post-operative case of gynecomastia grade 3 .

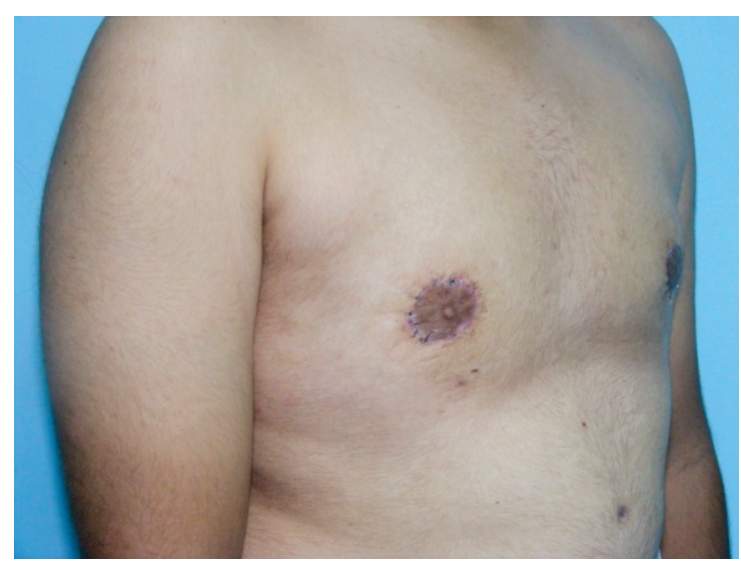

Fig. (2B): Post-operative case of gynecomastia grade 3 .

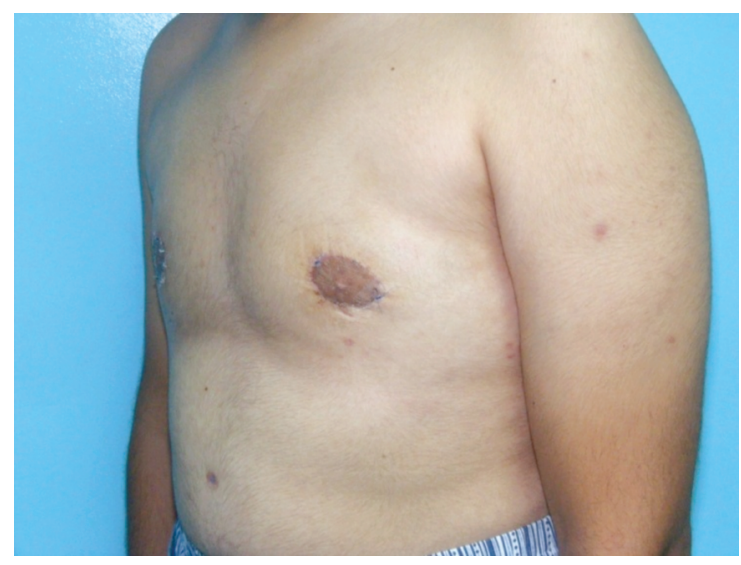

Fig. (3B): Post-operative case of gynecomastia grade 3 .

- 2b: Liposuction and use chest garment for 6-12 months before skin excision.

\section{Fibrous Gynecomastia:}

-1, 2a: Peri areolar skin incision and pull through tissue resection or ultrasonic assisted liposuction.

- 2b: Open approach through Peri areolar skin incision and resection and garment or ultrasonic 
assisted liposuction and pull through tissue resection.

- Grade 3: Skin resection through superior, inferior, concentric, omega, nipple transposition and liposuction and pull through tissue resection and garment usage.

Complications from previously mentioned techniques varied from Hematoma, under resection, over resection (saucer-type deformity), infection, Widening of scar and Asymmetry.

We are introducing a modification and an addition to the above-mentioned techniques and we named it: Quadruple attack to the problem of gynecomastia.

\section{Conclusion:}

Using suction lipectomy alone for grade II, III gynecomastia may not reach the desirable aesthetic results for patient and doctor. Quadruple attack is a new surgical technique which is simple, reliable and easily attained by young surgeons. Nevertheless, it has good results with less complication rate.

\section{REFERENCES}

1- Nydick M., et al.: Gynecomastia in adolescent boys. JAMA, 178: 449, 1961.

2- Williams M.J.: Gynecomastia: its incidence, recognition and host characterization in 447 autopsy cases. Am. J. Med., 34: 103, 1963.

3- Nuttal F.Q.: Gynecomastia as a physical finding in normal men. J. Clin. Endocrinal. Metab., 48: 338, 1979.

4- Simon G.E., Hoffman S. and Kahn S.: Classification and surgical correction of gynecomastia. Plast. Reconstr. Surg., 51: 48, 1973.

5- Rohrich R.J., Ha R.Y., Kenkel J.M., et al.: Classification and management of gynecomastia: Defining the roleof ultrasound-assisted liposuction. Plast. Reconstr. Surg., 111: 909, 2003.

6- Letterman G. and Schurter M.: Gynecomastia. In: Georgiade NG, ed. Reconstructive Breast Surgery. St. Louis, Mo: Mosby, 229-53, 1976.

7- Letterman G. and Schurter M.: Gynecomastia. In: Courtiss EH, ed. Male Aesthetic Surgery. St. Louis, Mo: Mosby; 295, 1982.

8- Letterman G. and Schurter M.: Surgical correction of massive gynecomastia. Plast. Reconstr. Surg., 49: 259, 1972. 